\title{
Concepciones Sobre Evaluación en la Formación Inicial del Profesorado de Ciencias, Tecnología y Matemáticas
}

\author{
Assessment's Conceptions of Pre-service Secondary Science, \\ Technology and Maths Teachers
}

\author{
Alfonso Pontes Pedrajasi* ${ }^{*}$, Francisco J. Poyato López ${ }^{1}$ y José Mª ${ }^{\text {a Oliva Martínez }}{ }^{2}$ \\ (1) Universidad de Córdoba y ${ }^{(2)}$ Universidad de Cádiz
}

\begin{abstract}
En este trabajo se muestran los resultados de un proyecto de innovación docente sobre evaluación educativa, desarrollado en el Máster de formación del profesorado de educación secundaria, aplicando una metodología de enseñanza basada en el enfoque reflexivo. Han participado, durante dos cursos académicos, 71 estudiantes de varias especialidades del área científico-técnica, realizando en el aula actividades que han permitido explicitar y debatir sus ideas previas sobre la evaluación del aprendizaje. Mediante un análisis de tipo cualitativo se han estudiado las opiniones de los participantes en torno a un conjunto de cuestiones abiertas, lo cual nos ha permitido conocer las concepciones de los futuros docentes sobre la evaluación, analizar su relación con diversos modelos didácticos y valorar el grado de consistencia de tales ideas. La experiencia ha resultado positiva porque nos ha permitido conocer mejor el pensamiento inicial de los participantes sobre la evaluación educativa. También nos sirve de estímulo para seguir trabajando en un proceso de formación en el que los futuros docentes realizan actividades orientadas a reflexionar sobre problemas de interés educativo, explicitando sus ideas previas y debatiendo sobre las diferentes concepciones como condición necesaria para impulsar la progresión del pensamiento profesional docente.
\end{abstract}

Palabras clave: Profesorado de enseñanza secundaria, Formación inicial, Concepciones previas, Evaluación educativa, Educación científico-técnica.

In this work, we show the results of a project of teaching innovation on educational evaluation, developed at the Master of Teacher Training Secondary Education, applying a methodology of teaching based on the reflexive approach. In this project, 71 students from various specialities of the scientific-technical area have participated during two academic courses. They have carried out activities that allow us to specify and discuss their previous ideas on assessment of learning. We have studied the opinions of the participants through a qualitative analysis of a set of open questions. This has enabled us to understand the conceptions of future teachers about the evaluation and, in addition, to analyze its relationship with various teaching models and to assess the degree of consistency of such ideas. The experience has been positive because it has permitted us to get a better understanding of the initial thinking of participants about the educational evaluation. It also encourage us to continue with this work, in which the future teachers put in practice activities aimed to reflect on issues of educational interest, describe their previous ideas and debate about the different conceptions, as a necessary condition to promote the progression of teacher professional thinking.

Keywords: Secondary education teachers, Initial training, Previous conceptions, Evaluation, Scientific and technical education.

*Contacto: apontes@uco.es issn: 1989-0397

www.rinace.net/riee/

https://revistas.uam.es/riee
Recibido: 28 de noviembre de 2015

$1^{\text {a }}$ Evaluación: 31 de enero de 2016 Aceptado: $\quad 28$ de febrero de 2016 


\section{Introducción ${ }^{1}$}

El desarrollo y puesta en marcha del llamado Espacio Europeo de Educación Superior (EEES) ha supuesto para la Universidad Española un proceso de transformación y convergencia de la enseñanza superior, que también ha afectado al curso de Formación Inicial del Profesorado de Secundaria (FIPS), ya que el anterior modelo de formación inicial, basado en el curso para la obtención del Certificado de Aptitud Pedagógica (CAP) presentaba numerosas limitaciones y había quedado obsoleto (Perales, 2014). Desde el curso 2009-10 la FIPS se ha integrado de forma oficial en los estudios universitarios de postgrado y se ha transformado en el Máster de Profesorado de Enseñanza Secundaria (MPES), donde se pretenden desarrollar competencias docentes de carácter profesional en un marco formativo más amplio y mejor organizado. Este hecho ha supuesto un importante cambio en la estructura del plan de estudios de la FIPS y en los programas de las materias de los diversos módulos teóricos y de la fase práctica, pero también debería aprovecharse para renovar los métodos de formación e impulsar la innovación y la investigación educativa en el campo de la formación docente (García-Carmona, 2013; Serrano y Pontes, 2015).

En este contexto han cobrado auge los trabajos orientados a mejorar el conocimiento de los profesores de secundaria en formación inicial, investigando sus motivaciones, actitudes, expectativas y, sobre todo, sus concepciones sobre los múltiples aspectos que configuran la profesión docente (e.g. Brown, Lake y Matters, 2011; Meirink, Meijer, Verloop y Bergen, 2009), con objeto de diseñar propuestas metodológicas que favorezcan el cambio de las ideas previas de los futuros docentes y les ayuden a construir conocimientos profesionales adecuados para desarrollar la actividad docente en los actuales centros de educación secundaria (Porlán y Martín, 2006; Sethusha, 2013). La finalidad principal de este trabajo consiste en plantear actividades que permitan a los futuros docentes de enseñanza secundaria reflexionar sobre la problemática de la evaluación del aprendizaje (De Wries, 2015; Turpo-Gebera, 2011; Vergara, 2012), permitiendo explicitar sus concepciones previas sobre este tema y favorecer el cambio conceptual (Abell, 2007; Hewson, 1993). Al mismo tiempo pretendemos recoger datos que nos permitan conocer las características de tales concepciones, por considerar que pueden ser útiles para el avance de la investigación sobre el pensamiento del profesorado (Carbajal y Gómez, 2002; Hernández, 2010).

\section{Fundamento y antecedentes}

\subsection{Modelos didácticos y concepciones del profesorado sobre los procesos educativos}

La práctica docente está influenciada en gran medida por el pensamiento de los profesores sobre los procesos educativos y para mejorar la calidad de la educación es necesario mejorar el proceso de formación del profesorado, teniendo en cuenta -entre

\footnotetext{
${ }^{1}$ Ayudas: Este trabajo forma parte de un proyecto de innovación y mejora de la calidad docente, desarrollado en sucesivas fases, contado con la financiación del Vicerrectorado de Planificación y Calidad Educativa de la Universidad de Córdoba (España).
} 
otros aspectos- el papel que desempeñan las concepciones personales en los procesos de formación (Abell, 2007; Mellado, Blanco y Ruiz, 1999; Porlán, Rivero y Martín del Pozo, 1997). Se han realizado muchas investigaciones orientadas a detectar las concepciones previas y creencias del profesorado, en formación inicial o en activo, utilizando diferentes tipos de instrumentos (Contreras, 2010; Valbuena, 2007) y en relación con los antecedentes de nuestra investigación presentan especial importancia los trabajos sobre el pensamiento docente que abordan problemas educativos de interés para los futuros docentes, desde un enfoque reflexivo (Fuentes, García y Martínez, 2009; García-Carmona, 2013; Pontes, Poyato y Oliva, 2015). También nos parecen interesantes aquellos estudios en los que se detectan dificultades de aprendizaje o los que permiten representar el cambio producido en las concepciones docentes durante la formación inicial (Solís, Porlán y Rivero, 2012). Consideramos que los formadores del profesorado pueden utilizar los resultados de tales investigaciones para promover la progresión de las ideas previas y ayudar a construir un conocimiento profesional adecuado (Dixon y Haigh, 2009).

En varios de los trabajos precedentes se han logrado identificar algunos perfiles característicos del pensamiento y la acción docente, que pueden interpretarse en términos de modelos didácticos diferenciados, ya que ofrecen una visión propia y distintiva de los procesos educativos en el área científico-técnica (Fuentes et al., 2009; Porlán et al., 1997; Pontes et al., 2015; Solís et al., 2012). Existe mucha literatura sobre el tema de los modelos didácticos y su relación con el pensamiento docente, pero hay trabajos de revisión bibliográfica que ofrecen una panorámica global e interesante sobre el tema (Hernández y Maquilón, 2010). En un trabajo de síntesis sobre los diferentes perfiles del profesorado de ciencias, llevado a cabo por Oliva (2008), se han caracterizado cuatro modelos didácticos que responden a las denominaciones genéricas siguientes: (1) Tradicional, (2) Tecnológico, (3) Activista y (4) Socio-constructivista. Los modelos 1 y 4 están muy diferenciados, pues representan visiones contrapuestas del pensamiento docente, ya que el modelo tradicional se identifica con un enfoque educativo basado en la transmisión-recepción mientras que el modelo socioconstructivista presenta un carácter innovador y se relaciona con un proceso de enseñanza-aprendizaje basado en la construcción de nuevas ideas a partir de los conocimientos previos. Sin embargo, los modelos 2 y 3 pueden considerarse como modelos intermedios o híbridos, ya que a veces incluyen características próximas a los modelos situados en los extremos de la escala anterior. Los estudios que permiten relacionar las concepciones previas del profesorado con los diferentes modelos didácticos que caracterizan el pensamiento docente nos parecen interesantes porque tales modelos permiten visualizar de forma más clara la progresión del conocimiento que se produce a través del proceso de formación (Fuentes et al., 2009, Valbuena, 2007).

En la línea de investigación sobre el pensamiento docente, hemos realizado anteriormente varios estudios sobre las concepciones de profesores en formación inicial, en torno a diversas estrategias y recursos educativos relacionados con el enfoque reflexivo (Pontes, Serrano, Muñoz y López, 2011) o la profesionalidad docente (Pontes, Serrano y Poyato, 2013). En un trabajo reciente hemos explorado las concepciones de profesores en formación sobre el aprendizaje de las ciencias (Pontes et al., 2015) y hemos encontrado que tales concepciones se agrupan mayoritariamente en torno a dos enfoques educativos diferenciados que hemos denominado "tradicional" e "innovador", aunque también hay muchas ideas de carácter dual o ambivalente que podrían formar parte de un enfoque intermedio. El enfoque innovador se aproxima 
bastante al modelo socio-constructivista, aunque los profesores en formación no presentan un esquema de ideas tan estructurado y coherente como el modelo didáctico representado bajo esta denominación (Contreras, 2010; Oliva, 2008), de modo que hemos optado por usar un término menos comprometido, aunque somos conscientes de que el enfoque innovador puede resultar un tanto ambiguo. En este nuevo estudio vamos a seguir avanzando en esta línea centrándonos en explorar las concepciones previas de los estudiantes del MPES sobre la evaluación del aprendizaje.

\subsection{Concepciones del profesorado acerca de la evaluación del aprendizaje}

De Vries (2015) ha analizado recientemente las múltiples facetas de la evaluación educativa y ha destacado la importancia del proceso de evaluación del aprendizaje para mejorar la calidad de la enseñanza, indicando que este tema debe ser tratado con cuidado por su complejidad siendo interesante impulsar la investigación en este campo. Uno de los aspectos a considerar, a la hora de mejorar el proceso de evaluación del aprendizaje, es el papel que desempeñan las concepciones, creencias y teorías implícitas del profesorado respecto de dicho proceso (Prieto y Contreras, 2008; Borko, 1997).

Desde hace tiempo se han realizado interesantes investigaciones sobre las concepciones del profesorado, en formación o en ejercicio, respecto a la evaluación del aprendizaje. Un trabajo pionero en España fue realizado por Buendía, Carmona, González y López (1999), quienes abordaron el estudio de las concepciones del profesorado de secundaria sobre la evaluación utilizando una batería de cuestiones abiertas. Tales autores ofrecen una síntesis interesante de las ideas de los docentes sobre la evaluación, que podríamos considerar bastante próximas al enfoque educativo tradicional porque hacen hincapié en términos tales como valorar, medir, sancionar o promocionar al alumnado, pero los profesores son conscientes de la importancia de la evaluación para mejorar los procesos de enseñanza-aprendizaje y algunos docentes también consideran que la evaluación es importante para hacer un seguimiento del proceso de construcción del conocimiento de los alumnos, lo cual se relaciona con un enfoque constructivista o innovador de la evaluación.

Otros autores como Segers y Tillema (2011) realizaron un estudio destinado a explorar las concepciones de profesores y alumnos de secundaria sobre la evaluación. Observaron que los alumnos no distinguen entre evaluación sumativa y formativa, pero los profesores sí hacen esa distinción. Los profesores tienen una visión más compleja pues consideran, entre otros aspectos, la responsabilidad de la escuela y del profesorado para mejorar la evaluación y, sobre todo, los efectos que la evaluación tiene en el proceso de aprendizaje. Por su parte, Remesal (2011) también ha realizado un estudio sobre las concepciones de profesores de secundaria y maestros acerca de la evaluación, elaborando un modelo de análisis de tales concepciones en el que se consideran cuatro dimensiones. Tales dimensiones permiten estudiar los efectos de la evaluación sobre la enseñanza, el aprendizaje, la responsabilidad del profesorado o de la escuela y la certificación de los resultados académicos alcanzados. El estudio puso de manifiesto la dificultad de poner en práctica un sistema de evaluación innovador y alternativo que ayude a mejorar el proceso de aprendizaje al profesorado de ambos niveles que participaba en el proyecto.

Monteagudo, Molina y Miralles (2015) han explorado recientemente las opiniones sobre la evaluación del profesorado español del área de ciencias sociales en la educación secundaria. Tales autores observan que los docentes en ejercicio muestran escasa formación en el ámbito evaluativo, y que continúan evaluando a los alumnos 
básicamente mediante procedimientos tradicionales, como los exámenes escritos que enfatizan contenidos conceptuales. Según los citados autores el enfoque de competencias básicas está poco presente en las concepciones del profesorado y concluyen que las reformas legales educativas tienen escasa repercusión en la práctica evaluadora cotidiana de los profesores de este nivel.

En relación al tema de las concepciones del profesorado Dixon y Haigh (2009) realizaron un estudio cualitativo donde se observa la influencia mediadora de las creencias de un conjunto reducido de profesores de matemáticas en torno al proceso de evaluación y se analizan los cambios en el pensamiento docente sobre los roles de profesores y alumnos en relación a dicho proceso. Por su parte, Wang, Kao y Lin (2010) describen y analizan las concepciones sobre el aprendizaje y la evaluación de la ciencia. Los resultados muestran que el modo de evaluar de los profesores en formación estaba más próximo a una visión tradicional del aprendizaje.

En un contexto más cercano a nuestra investigación, Hernández (2010) ha realizado un estudio de las concepciones sobre evaluación de profesores de ciencias de secundaria en formación inicial, en el curso del CAP antes citado, utilizando una batería de cuestiones abiertas donde se abordan tópicos clásicos sobre el tema ya analizados por Buendía et al. (1999), aunque en este caso los participantes tenían que responder desde dos puntos de vista: según su experiencia real como alumnos y según su idea de cómo debería ser el proceso de evaluación. Entre las implicaciones educativas de este trabajo se destaca la necesidad de ayudar a los profesores en formación a reflexionar sobre la evaluación del aprendizaje desde una perspectiva formativa y motivadora. Por su parte, TurpoGebera (2011) ha analizado las concepciones y prácticas evaluativas de los docentes de secundaria del área de ciencias, usando un cuestionario como instrumento de aproximación al pensamiento docente. El autor indica que, a pesar de los procesos de formación y capacitación docente, subsisten intervenciones tradicionales en las formas y modos de evaluar los aprendizajes. En este estudio también se observa que, a través de las cuestiones planteadas, afloran posicionamientos relativamente innovadores sobre la evaluación, aunque en conjunto revelan situaciones de incertidumbre y ambivalencia.

En el ámbito anglosajón, Brown et al. (2011) han investigado las diferentes creencias sobre la evaluación del aprendizaje de las ciencias, entre profesores de enseñanza secundaria y primaria, poniendo de manifiesto la importante relación entre las creencias de los docentes y las diferentes prácticas de evaluación en ambos niveles de enseñanza. Los resultados mostraron que los maestros de primaria están más de acuerdo que los profesores de secundaria con la idea de que el sistema de evaluación usado por cada profesor determina la calidad del proceso de enseñanza y la mejora del aprendizaje. Así mismo Halinen, Ruohoniemi, Katajavuori y Virtanen (2014) han estudiado las concepciones sobre la evaluación en profesores de biología y han identificado tres categorías o modelos docentes atendiendo al discurso de cada profesor sobre el tema. Tales autores consideran que la formación pedagógica, la relación con los compañeros, el apoyo institucional y la interacción con la comunidad educativa influyen de diferente manera en el sistema de evaluación que cada docente realiza en la práctica. También indican que estas variables deben considerarse cuidadosamente a la hora de implementar procesos formativos que contribuyan a mejorar los sistemas de evaluación del profesorado.

De la revisión de la literatura realizada se deriva que los profesores en formación y en activo tienen ideas diferentes sobre la forma de evaluar, que están relacionadas con su propia visión acerca de la enseñanza y el aprendizaje. En los profesores en activo 
influye mucho su experiencia docente y el contexto educativo donde actúan, mientras que en los profesores en formación las concepciones previas parecen estar más influenciadas por el recuerdo de cómo han sido evaluados cuando eran alumnos (Hernández, 2010). Aunque hay muchos antecedentes sobre esta temática observamos que la mayoría de los estudios revisados se han realizado en el extranjero o en un contexto diferente al nuestro, siendo todavía escasos los estudios sobre este tema desarrollados en el marco que ofrece el máster de profesorado de secundaria. También echamos de menos investigaciones que profundicen en la relación entre concepciones previas sobre los diversos procesos educativos y los modelos didácticos subyacentes a tales concepciones (Solís et al., 2012) o estudios que aporten datos sobre las fortalezas y debilidades del proceso de formación desarrollado en cada experiencia (Perales, 2014). En este nuevo contexto nos parece necesario desarrollar estudios sobre la evaluación del aprendizaje que traten de llevar a la práctica el enfoque reflexivo en la formación inicial docente (García Carmona 2013), describiendo el tipo de actividades que se realizan en el aula y analizando las concepciones de los estudiantes del máster que salen a la luz en el desarrollo de tales actividades. La necesidad de tener en cuenta tales aspectos constituye la justificación y el principal interés del presente estudio.

\section{Diseño de la investigación}

En esta investigación se ha adoptado un enfoque metodológico mixto, basado en un análisis cualitativo y cuantitativo de los datos recogidos. En primer lugar intentamos acercarnos al conocimiento de las ideas del alumnado mediante técnicas de análisis cualitativo (Rodríguez, Gil y García, 1996) y en segundo lugar se realiza un análisis cuantitativo de frecuencias y porcentajes correspondientes a las diversas categorías de ideas recogidas en la investigación. Se trata, por tanto, de un estudio descriptivo, basado en un diseño no experimental.

\subsection{Objetivos}

El estudio se enmarca en un proyecto de investigación más amplio cuyo contexto, finalidad global y resultados previos se han expuesto en varios trabajos anteriores relacionados con las concepciones de los futuros docentes sobre los procesos de enseñanza y aprendizaje (Pontes et al., 2013, 2015). En esta ocasión nos centraremos en el análisis del pensamiento docente de los estudiantes del MPES sobre la evaluación, persiguiendo los objetivos específicos siguientes:

$\checkmark$ Explorar las concepciones previas de los estudiantes del MPES sobre la evaluación del aprendizaje de la ciencia.

$\checkmark$ Analizar sus ideas sobre los recursos e instrumentos de evaluación que consideran más importantes para valorar el proceso de aprendizaje del alumnado de secundaria.

$\checkmark$ Estudiar el grado de coherencia interna o consistencia inter-contextual de los modelos didácticos subyacentes en las concepciones previas de los participantes sobre diversos aspectos de la evaluación.

\subsection{Participantes}

En esta experiencia formativa han participado 71 estudiantes del MPES de diversas especialidades del área científico-técnica, que han cursado el citado máster en la Universidad de Córdoba (España), entre los cursos académicos 2009-10 y 2010-11, de 
los que 39 eran mujeres y 32 eran hombres, con una edad media de 26.5 años. Por especialidades han participado 2 grupos de Biología, Salud y Deporte (28 estudiantes), 2 grupos de Física-Química (19 estudiantes), 1 grupo de Tecnología (13 estudiantes) y 1 grupo de Matemáticas e Informática (11 estudiantes). Algo menos de una quinta parte de tales estudiantes han tenido experiencia docente y todos han expresado su deseo de cursar el máster de profesorado por varias razones analizadas en un estudio previo: algo más de la mitad por tener acceso laboral a centros docentes públicos y privados, una cuarta parte han mostrado un interés de tipo vocacional por la docencia y el resto alude a razones de otro tipo, como ampliar el currículum académico o ampliar el campo de posibles salidas profesionales. En cualquier caso todos los participantes están bastante interesados en adquirir una buena formación pedagógica y didáctica, sobre todo en aspectos metodológicos, comunicativos y en el uso de recursos docentes (Pontes y Poyato, 2014).

\subsection{Recogida y análisis de datos}

La recogida de datos de este estudio se realizó en una clase del módulo específico del máster, utilizando una batería de cuestiones abiertas que formaban parte del programa de actividades de aula y que se exponen en el cuadro 1. Para ello hemos contado con la colaboración de diversos docentes de la asignatura "Aprendizaje y Enseñanza de las Materias de la Especialidad (AEME)", que han utilizado el material didáctico elaborado para este proyecto. Estas cuestiones se han planteado antes de iniciar el bloque de contenidos sobre evaluación incluido en dicha materia, de modo que las concepciones registradas en este estudio pueden considerarse como ideas previas de los estudiantes del MPES, aunque tales ideas pueden estar influenciadas por los conocimientos adquiridos en las materias de formación psicopedagógica cursadas en el módulo genérico del máster.

A lo largo del proyecto se ha aplicado la misma metodología de análisis cualitativo empleada en trabajos anteriores (Pontes et al., 2015), basada en la separación y la clasificación de ideas primarias en diferentes categorías y subcategorías a partir de las respuestas de los estudiantes en cada cuestión (Rodríguez et al., 1996; Buendía et al., 1999; Hernández, 2010). En esta fase de análisis han intervenido tres investigadores expertos en didáctica de las ciencias y formación del profesorado, adoptando criterios comunes a la hora de categorizar y clasificar las ideas básicas que forman parte de cada respuesta. Este método de trabajo es dificultoso y no está exento de contradicciones, pero por coherencia metodológica hemos preferido mantener el mismo enfoque en el tratamiento de los datos recogidos en esta primera fase del proyecto. En una etapa posterior hemos comenzado a utilizar el software de análisis cualitativo "Atlas.ti" al explorar otros aspectos del pensamiento docente relacionados con el desarrollo de la identidad profesional (Serrano y Pontes, 2016). Tras el proceso de categorización anterior se han contabilizado las ideas básicas que forman parte de cada respuesta y se ha realizado un análisis de frecuencias y porcentajes que se incluyen en cada una de las tablas de ideas registradas en las diferentes cuestiones. Por limitaciones de espacio, en este estudio sólo se analizarán los resultados correspondientes a las actividades A3 y A4, que son las que han proporcionado un mayor número de ideas interesantes sobre la evaluación, por parte de los participantes.

En tales cuestiones se han analizado sus opiniones y se han registrado las ideas expresadas individualmente por escrito, encontrando en todos los casos un número mayor de ideas básicas (y relativamente diferenciadas dentro de una respuesta más o 
menos amplia) que el número de sujetos encuestados, porque cada estudiante ha expresado por término medio más de una idea diferente en cada cuestión. Por ello el análisis de frecuencias relativas o porcentajes que se mostrará en la sección de resultados se realizará sobre el cómputo total de ideas diferentes registradas en cada cuestión. Sin embargo, a la hora de realizar un estudio de consistencia de los modelos didácticos subyacentes en tales concepciones se utilizará sólo la idea principal expresada por cada sujeto en ambas cuestiones, de modo que en dicho análisis los porcentajes se refieren al número de ideas principales, que a su vez coinciden con el número de participantes en la experiencia.

A1. Cita por orden de importancia las principales competencias (máximo 50 6) que debe adquirir el profesorado de enseñanza secundaria de tu especialidad durante el proceso de formación inicial, incluyendo si procede la competencia evaluadora (saber evaluar).

A2. ¿Cuáles son los principales fines o funciones de la evaluación educativa en el área de ciencias y en el contexto de la educación secundaria?

A3. ¿En qué consiste la evaluación educativa y qué relación existe entre evaluación del aprendizaje y mejora de la enseñanza?

A4. ¿Qué recursos o qué o instrumentos de evaluación crees que son los más importantes para valorar el proceso de aprendizaje del alumnado en materias de tu especialidad

A5. ¿Cómo y cuando es necesario evaluar? Indicar posibles etapas del proceso de evaluación comentando sus características

A6. ¿Quiénes son los agentes del proceso de evaluación? ¿Es necesario evaluar también el proceso de enseñanza? ¿Por qué motivos y cómo hacerlo?

Figura 1: Cuestiones abiertas sobre la evaluación

Fuente: Elaboración propia.

\section{Resultados}

En primer lugar se analizan las principales concepciones previas de los participantes sobre las cuestiones planteadas en las actividades A3 y A4 del programa-guía recogido en el Cuadro 1, mostrando las tablas de resultados que se han obtenido en tales cuestiones, tras el análisis de las respuestas correspondientes a cada cuestión y la categorización de las ideas registradas en cada pregunta. Después de cada tabla se incluyen y comentan ejemplos de algunos tipos de respuestas representativas en cada categoría de ideas. Al tratarse de cuestiones generales hemos observado ciertas pautas o tendencias que son comunes a los estudiantes de diferentes especialidades (Biología, Física, Matemáticas o Tecnología), y son tales pautas las que se reflejan principalmente en los datos cuantitativos que se muestran en las tablas posteriores. Sin embargo, al tratar de analizar las ideas sobre evaluación en conexión con los modelos didácticos subyacentes del alumnado del MPES, hemos sido conscientes de que puede haber conexión entre tales modelos y las concepciones epistemológicas de los estudiantes, que a su vez dependen de la formación académica previa y del conocimiento didáctico del contenido de la propia disciplina (Valbuena, 2007). Por ello creemos que sería conveniente realizar estudios posteriores más específicos, que permitan profundizar en este asunto, ya que en este estudio sólo se han abordado aspectos muy generales del pensamiento docente sobre la evaluación. 


\subsection{La evaluación educativa y su relación con la mejora de la enseñanza}

En la cuestión planteada en la actividad A3 los futuros docentes deben explicar en qué consiste la evaluación educativa y qué relación existe entre evaluación y mejora de la educación científico-técnica. Se han analizado las opiniones de los participantes sobre esta temática y se han registrado 134 ideas sobre este tema, lo cual supone una media de 1,9 por sujeto, identificando diferentes categorías y sub-categorías de opiniones, que han permitido realizar un estudio de frecuencias y porcentajes, cuyos datos se muestran en la tabla 1 y se comentan posteriormente.

En la categoría A se recogen opiniones sobre la evaluación que podemos considerar próximas al enfoque educativo tradicional según la clasificación propuesta por diversos autores (Porlán et al., 1997), ya que se identifica principalmente con aspectos como la medida de los conocimientos adquiridos por los alumnos, lo cual se refleja en una nota de examen o calificación numérica y que, en la práctica, actúa como un mecanismo de selección o de promoción. En esta categoría se incluye algo más de una tercera parte de las ideas registradas. Por ejemplo:

La evaluación supone medir el nivel de conocimientos alcanzado por los alumnos respecto a los objetivos previstos". "En la enseñanza secundaria no es posible llevar un seguimiento individual del aprendizaje de cada alumno, de modo que hay que hacer exámenes escritos, porque de lo contrario los alumnos no estudiarían la asignatura.

En la categoría B se incluyen opiniones que recogen una visión más integral de la evaluación y que podemos considerar próximas al modelo educativo socioconstructivista (Fuentes et al., 2009). Así, se asume que la evaluación supone un proceso en el que se pueden recoger múltiples datos sobre el proceso de aprendizaje y que tales datos sirven de instrumento para la reflexión del profesorado y para favorecer la mejora de la acción docente. En esta categoría se incluye algo menos de una tercera parte de las respuestas analizadas. Por ejemplo:

La evaluación es el proceso mediante el cual se analiza el grado de consecución de las metas educativas que te has propuesto en la asignatura. La relación con la mejora de la enseñanza es evidente porque la evaluación siempre se realiza con la intención de mejorar el proceso de enseñanza y aprendizaje, de conocer dónde hay deficiencias para resolverlas o si es necesario cambiar la metodología, etc.

En la categoría C se recogen otras opiniones sobre la evaluación que podríamos considerar en una zona intermedia entre el enfoque tradicional y el enfoque socioconstructivista, en la que se integran algo más de una cuarta parte de las ideas registradas:

Evaluar supone una valoración del alumnado en la adquisición de los conocimientos mínimos. Pero la mayoría de veces nos centramos en qué se enseña y no en cómo se enseña, ahí es donde está la clave para mejorar. Siempre es necesario evaluar el nivel que adquieren los alumnos mediante exámenes. Pero también es importante indagar lo que de verdad aprenden los alumnos y reflexionar sobre aquello que favorece la mejora de la educación.

Como ocurría en otras cuestiones analizadas en este proyecto relacionadas con el aprendizaje en materias del área científico-técnica (Pontes et al, 2015), también en torno a la evaluación aparecen numerosas concepciones próximas al enfoque educativo tradicional (A), pero es interesante que haya casi un tercio de opiniones de carácter innovador (B), próximas al enfoque constructivista (Solís et al., 2012). Por otra parte observamos que en la posición intermedia o ambivalente (B) subyace la idea de que la 
evaluación es un proceso de valoración del rendimiento escolar del alumno, pero también se incorporan otras ideas próximas al enfoque innovador como son la valoración de competencias y destrezas, la evaluación de conocimientos previos para valorar el avance del alumno o la posibilidad de incorporar a los alumnos al proceso de evaluación, como complemento a la valoración del profesor.

Tabla 1. Concepciones sobre la evaluación educativa

\begin{tabular}{|c|c|c|}
\hline TIPOS DE IDEAS REGISTRADAS & FREC. & $\%$ \\
\hline (A) Enfoque tradicional: & 46 & $(34,3)$ \\
\hline - La evaluación como medida de conocimientos adquiridos & 24 & 17,9 \\
\hline - La evaluación como sinónimo de nota o calificación & 16 & 11,9 \\
\hline - La evaluación como mecanismo de selección & 6 & 4,5 \\
\hline (B) Enfoque innovador: & 42 & $(31,4)$ \\
\hline $\begin{array}{l}\text { - La evaluación como recogida de múltiples datos sobre el } \\
\text { proceso de aprendizaje (conocimientos, actitudes, destrezas, } \\
\text { esfuerzo,...) }\end{array}$ & 21 & 15,7 \\
\hline - La evaluación como instrumento de reflexión del profesorado & 12 & 9,0 \\
\hline $\begin{array}{l}\text { - La evaluación como recurso de indagación y mejora de la acción } \\
\text { docente }\end{array}$ & 9 & 6,7 \\
\hline (C) Enfoque intermedio & 37 & $(27,6)$ \\
\hline $\begin{array}{l}\text { - Medir conocimientos adquiridos } y \text { otros aspectos } \\
(\text { competencias, destrezas,...) }\end{array}$ & 19 & 14,2 \\
\hline $\begin{array}{l}\text { - Medir para comprobar el rendimiento del proceso educativo, } \\
\text { analizando lo que saben los alumnos antes y después. }\end{array}$ & 8 & 5,9 \\
\hline - Coherencia entre método de enseñanza y sistema de evaluación & 6 & 4,5 \\
\hline $\begin{array}{l}\text { - Incorporar a los alumnos al proceso de evaluación, como } \\
\text { complemento a la valoración del profesor }\end{array}$ & 4 & 3,0 \\
\hline (D) Otras ideas poco explícitas (difíciles de categorizar) & 8 & $(5,9)$ \\
\hline
\end{tabular}

Fuente: Elaboración propia.

\subsection{Recursos e instrumentos para evaluar el aprendizaje en materias de la especialidad}

En la cuestión planteada en la actividad A4 se solicita a los estudiantes del máster FPES que expongan sus ideas sobre los recursos o los instrumentos de evaluación que consideran más importantes para valorar el proceso de aprendizaje del alumnado de secundaria en materias del área científico-técnica. Tras analizar las 124 respuestas distintas encontradas para esta cuestión ( 1,7 respuestas por alumno), se han identificado diferentes categorías y se ha realizado un estudio de frecuencias y porcentajes, cuyos datos se muestran en la tabla 2 y se comentan a continuación.

En los resultados correspondientes a esta cuestión también se recogen en la categoría A las opiniones sobre los recursos e instrumentos de evaluación relacionadas con el enfoque educativo tradicional (Oliva, 2008), ya que el principal medio utilizado para medir el rendimiento académico es el examen (aunque incluya diversos tipos de preguntas tales como cuestiones, ejercicios, etc.) y en otros casos se mantiene el examen como recurso básico para obtener una calificación, pero se complementa (de forma vaga e imprecisa) con otro tipo de datos: la participación en clase, las tareas realizadas en casa, etc. En esta categoría se incluye alrededor de una sexta parte de las ideas registradas sobre este tema. Por ejemplo:

La evaluación está orientada a medir los conocimientos adquiridos por cada alumno y, por tanto, creo que el examen escrito (incluyendo teoría y problemas) debe ser el principal recurso. Veo difícil que en asignaturas como la biología se puedan sustituir los exámenes 
por otro tipo de instrumentos de evaluación, pero se puede complementar la nota del examen con otros datos.

En la categoría B se incluyen ideas sobre la forma de llevar a cabo la evaluación que podríamos considerar próximas al modelo educativo constructivista, porque en muchos casos se asume la evaluación como un proceso continuo e integral, que requiere el uso de una amplia gama de recursos e instrumentos destinados a evaluar diferentes aspectos del proceso de aprendizaje (conocimientos teóricos, destrezas de tipo práctico, capacidad de análisis y resolución de problemas, capacidad de relacionar conceptos, actitud en clase, participación y capacidad de trabajar en equipo). Se concede importancia a los conocimientos previos y a la observación del trabajo realizado por el alumno en el aula, o se habla de registrar tales observaciones en el diario del profesor. Pese a las dificultades de este modelo de evaluación, encontramos que más dos quintas partes de las ideas registradas pueden incluirse en esta categoría. Por ejemplo:

El aprendizaje de las ciencias es un proceso complejo y se deben usar instrumentos de evaluación variados y complementarios como explicación de hechos, resolución de problemas, mapas conceptuales...En Biología es importante saber evaluar las competencias generales y específicas de la materia, las destrezas mostradas en el aula taller al desarrollar proyectos, la actitud del alumno en el aula y también hay que evaluar los conocimientos adquiridos mediante diferentes tipos de pruebas escritas.

Tabla 2. Ideas sobre recursos e instrumentos de evaluación

\begin{tabular}{lcc}
\hline \multicolumn{1}{c}{ TIPOS DE IDEAS REGISTRADAS } & FREC. & (\%) \\
\hline (A) Evaluación orientada a medir conocimientos & 20 & $(16,1)$ \\
- El examen escrito como principal recurso & 11 & $(8,9)$ \\
- Complementar la nota de exámenes con otros datos & 9 & $(7,2)$ \\
(B) La evaluación como un proceso continuo e integral & 53 & $(42,7)$ \\
- Uso de instrumentos de evaluación variados & 26 & 20,1 \\
- Evaluación continua del trabajo del aula y del aprendizaje desarrollado & 15 & $(12,1)$ \\
- Evaluación de competencias, destrezas, actitudes y conocimientos & 12 & $(9,8)$ \\
(C) Posición ambivalente & 42 & $(33,9)$ \\
- Importancia de evaluar aspectos prácticos & 27 & $(21.8)$ \\
- Utilizar las nuevas tecnologías & 10 & $(8,1)$ \\
- Incluir elementos de autoevaluación o coevaluación & 5 & $(4,0)$ \\
(D) Otras ideas poco explícitas (difíciles de categorizar) & 9 & $(7,3)$ \\
\hline
\end{tabular}

Fuente: Elaboración propia.

De igual forma que en la cuestión anterior, también se recogen en la categoría C otras opiniones, sobre los instrumentos de evaluación, que podrían considerarse como visiones ambivalentes o intermedias entre el modelo tradicional y el enfoque innovador. Tales opiniones conceden importancia al examen escrito como instrumento de medida del rendimiento del alumno, pero consideran necesario complementar este aspecto usando otros recursos como son las nuevas tecnologías, las prácticas de laboratorio, el desarrollo de proyectos tecnológicos o la realización de actividades de autoevaluación y coevaluación. En esta categoría hemos registrado algo más de una tercera parte de las ideas mostradas por los futuros profesores del área de ciencias:

\footnotetext{
El examen clásico es un recurso importante para evaluar el aprendizaje de cada alumno, pero se deben tener en cuenta otros datos recogidos mediante el diario del profesor o la observación del trabajo experimental de los alumnos para evaluar la parte práctica de la asignatura". "En ciencias como la fisica y química hay que hacer muchos ejercicios y prácticas, fomentando la participación porque así resulta más fácil aprender, con lo cual se evaluará no solo algo escrito sino también el progreso realizado día a día.
}

En torno a esta cuestión también aparecen diversas opiniones difíciles de categorizar (7,3\%), porque algunos de los futuros profesores emiten respuestas muy vagas ("Me 
bastaría con inculcar una curiosidad científica") sobre la forma en la que se podría llevar a cabo el proceso de evaluación en sus respectivas materias.

\subsection{Consistencia de los modelos didácticos subyacentes en las concepciones previas de los futuros docentes de ciencias sobre la evaluación}

En la cuestión planteada en la actividad A3 (tabla 1) los futuros docentes deben explicar en qué consiste la evaluación educativa y qué relación existe entre evaluación y mejora de la educación científico-técnica. Por otra parte, en la cuestión planteada en la actividad A4 (tabla 2) se solicita a los estudiantes del máster FPES que expongan sus ideas sobre los recursos o los instrumentos de evaluación que consideran más importantes para valorar el proceso de aprendizaje del alumnado de secundaria en las materias del área.

Sobre ambas cuestiones se han recogido diversas ideas que se han clasificado con arreglo a dos modelos de pensamiento docente bien diferenciados como son el enfoque tradicional (A) y el enfoque innovador o constructivista (B). El primero se caracteriza por una visión de los procesos educativos que pone su acento en el protagonismo del profesor y los contenidos de la enseñanza, mientras que en el segundo cobra gran importancia el protagonismo del alumno en el aprendizaje, el papel de las actividades el proceso de enseñanza y la necesidad de llevar a cabo una evaluación acorde con la visión del aprendizaje y la enseñanza. Así mismo, se han encontrado en ambas cuestiones un número importante de ideas algo menos definidas, que presentan un carácter dual o ambivalente entre los enfoques extremos (A y B) y que se han incluido en el llamado enfoque intermedio (C). En ambos casos también hay ideas muy confusas, indefinidas o difíciles de clasificar en alguno de los modelos descritos anteriormente y que hemos incluido en el grupo $\mathrm{D}$, que es bastante minoritario.

Para ver hasta qué punto existe coherencia interna en los puntos de vista de los participantes sobre tales cuestiones, o en qué medida son consistentes los modelos didácticos subyacentes a las concepciones de los futuros docentes sobre la evaluación, se ha realizado un estudio cruzado de los resultados de ambas cuestiones utilizando un análisis basado en tablas de contingencia, incluyendo algunos estadísticos que miden el grado de relación entre los modelos cruzados y cuyos resultados se muestran en la tabla 3. Conviene recordar un aspecto señalado anteriormente y es que en ambas cuestiones se ha registrado, por término medio, más de una idea diferente y, por tanto, los porcentajes expresados en las tablas 1 y 2 se referían al número completo de ideas de cada cuestión, que es mayor que el número de sujetos de la muestra. Sin embargo, para estudiar la consistencia de los modelos didácticos subyacentes en las concepciones sobre evaluación, correspondientes a las dos cuestiones analizadas en este trabajo, se ha utilizado sólo la idea principal expresada por cada sujeto en ambas cuestiones. Por tanto, las frecuencias y porcentajes que se muestran en la tabla 3 se refieren tanto al número de ideas principales como al número de estudiantes, que en este caso son coincidentes.

En los resultados mostrados en la tabla 3 se puede observar que hay cierta relación de correspondencia entre los modelos de pensamiento de carácter tradicional (A), innovador (B) e intermedio (C) subyacentes a las principales concepciones de los futuros docentes sobre la evaluación del aprendizaje de las ciencias, registradas en las actividades A3 y A4. Lo cual indica que los estudiantes del MPES se identifican en cierta medida con alguno de tales enfoques didácticos y tienden a expresar ideas relativamente consistentes con su modelo de pensamiento en cuestiones diferentes. Sin 
embargo, los parámetros estadísticos que miden el grado de relación de tales modelos en las dos cuestiones analizadas no son suficientemente significativos para considerar que este resultado es del todo afirmativo. Creemos que ello puede ser debido a las limitaciones propias de los análisis de tipo cualitativo y, por tanto, consideramos necesario realizar nuevos estudios sobre esta temática en los que se utilicen instrumentos y técnicas de investigación basados en un enfoque cuantitativo.

Tabla 3. Tabla de contingencia entre modelos didácticos registrados en las actividades A3 y A4

\begin{tabular}{|c|c|c|c|c|c|c|}
\hline \multirow{2}{*}{\multicolumn{2}{|c|}{$\begin{array}{c}\text { MODELOS } \\
\text { DOCENTES EN A3 }\end{array}$}} & \multicolumn{4}{|c|}{ MODELOS DOCENTES EN A4 } & \multirow{3}{*}{$\begin{array}{c}\text { TotaL } \\
24\end{array}$} \\
\hline & & \multirow{2}{*}{$\begin{array}{c}\text { A } \\
6\end{array}$} & \multirow{2}{*}{$\begin{array}{c}\text { B } \\
13\end{array}$} & \multirow{2}{*}{$\frac{\mathbf{C}}{5}$} & \multirow{2}{*}{$\frac{\mathbf{D}}{\mathrm{O}}$} & \\
\hline $\bar{A}$ & Frec. & & & & & \\
\hline & $(\%)$ & 50,0 & 41,9 & 21,7 & 0,0 & 33,8 \\
\hline \multirow[t]{2}{*}{$\bar{B}$} & Frec. & 4 & 11 & 5 & 2 & 22 \\
\hline & $(\%)$ & 33,3 & 35,5 & 21,7 & 40,0 & 31,0 \\
\hline \multirow[t]{2}{*}{$\overline{\mathrm{C}}$} & Frec. & 1 & 7 & 11 & 2 & 21 \\
\hline & $(\%)$ & 8,3 & 22,6 & 47,8 & 40,0 & 29,6 \\
\hline \multirow[t]{2}{*}{$\overline{\mathrm{D}}$} & Frec. & 1 & $\mathrm{O}$ & 2 & 1 & 4 \\
\hline & $(\%)$ & 8,3 & 0,0 & 8,7 & 20,0 & 5,6 \\
\hline \multirow[t]{2}{*}{ Total } & Frec. & 12 & 31 & 23 & 5 & 71 \\
\hline & $(\%)$ & 16,9 & 43,7 & 32,4 & 7,0 & 100,0 \\
\hline
\end{tabular}

Notas: Razón de verosimilitudes: 17,453 (p: 0,046)

Tau-b de Kendall: 0,322 (p: 0,094)

Fuente: Elaboración propia.

\section{Discusión y conclusiones}

En este trabajo hemos mostrado los resultados de una experiencia educativa realizada durante el proceso de formación inicial del profesorado de secundaria, en diversas especialidades del área científico-técnica, tratando de poner en práctica el enfoque reflexivo en la formación docente (Hewson, 1993; García Carmona, 2013) y realizando en el aula actividades que permiten explicitar las ideas previas sobre la evaluación del aprendizaje (Buendía et al., 1999; Segers y Tillema, 2011). En primer lugar se han analizado las concepciones de los participantes sobre la evaluación del aprendizaje y sus ideas sobre los recursos e instrumentos de evaluación que consideran más importantes para valorar el proceso de aprendizaje del alumnado de secundaria. También hemos tratado de relacionar tales ideas con los modelos didácticos que presentan mayor consenso en la literatura sobre el pensamiento del profesorado (Oliva, 2008; Hernández y Maquilón, 2010; Solís et al., 2012), decantándonos por una clasificación de ideas que se mueve en torno a tres categorías principales: un modelo tradicional de enseñanza y evaluación, bien diferenciado de un modelo didáctico que hemos denominado innovador y que se encuentra próximo a la perspectiva socio-constructivista, existiendo entre ambos polos un enfoque intermedio de carácter dual o ambivalente, hecho que también pudimos constatar al analizar las concepciones de los futuros docentes sobre los procesos de aprendizaje (Pontes et al., 2015).

En la primera cuestión analizada hemos encontrado una proporción similar de concepciones previas sobre la evaluación que corresponden, respectivamente, al enfoque educativo tradicional, al enfoque innovador y al enfoque intermedio o dual. Sin embargo, en la segunda cuestión, al analizar las ideas sobre el uso de recursos e instrumentos, encontramos una proporción mayor de ideas innovadoras que ideas 
tradicionales. Nos parece interesante que muchos de los participantes muestren opiniones sobre la manera concreta de evaluar el aprendizaje de la ciencia que resultan próximas a posiciones constructivistas o alternativas (Porlán et al., 1997), aunque también hay un porcentaje importante de ideas de tipo tradicional. Nos parece necesario, por tanto, seguir avanzando y profundizando en el diseño de procesos formativos orientados a fomentar una visión de la evaluación de carácter integral que tenga en cuenta, además de los conocimientos adquiridos por los alumnos, la actitud hacia el aprendizaje, las destrezas o competencias adquiridas, el esfuerzo realizado y el comportamiento en aula. De esta forma se podría conseguir que las ideas sobre la evaluación de carácter tradicional se transformen en concepciones coherentes con un modelo didáctico constructivista e innovador (Solís et al., 2012).

En segundo lugar, pese a tratarse esencialmente de una investigación de carácter exploratorio y cualitativo, hemos tratado de estudiar también el grado de coherencia interna o consistencia inter-contextual de los modelos didácticos subyacentes en las concepciones previas de los participantes sobre los aspectos analizados anteriormente. El hecho de encontrar porcentajes diferentes entre las ideas de corte tradicional e innovador sobre la evaluación en función del tipo de cuestión planteada, según se aprecia en las tablas 1 y 2 , indica que las concepciones sobre la evaluación de los futuros docentes no son del todo consistentes o que dependen del contexto del problema (Wang et al., 2010). Por ello hemos introducido algunas técnicas propias del análisis cuantitativo para realizar un estudio cruzado de los resultados de las dos primeras cuestiones, utilizando un análisis basado en tablas de contingencia, cuyos resultados no pueden considerarse concluyentes por las limitaciones metodológicas que se han comentado al exponer los resultados mostrados en la tabla 3. En efecto, hemos observado que hay cierta relación de correspondencia entre los modelos de pensamiento de carácter tradicional, innovador e intermedio sobre la evaluación del aprendizaje, pero los parámetros estadísticos que miden el grado de relación de tales modelos no son suficientemente significativos, lo cual puede deberse al hecho de que tales resultados proceden de cuestiones abiertas relativamente diferentes y a las limitaciones propias del análisis cualitativo (Dixon y Haigh, 2009). También conviene considerar que las ideas sobre evaluación analizadas en este estudio corresponden al nivel declarativo cuya categorización resulta en ocasiones difícil (Valbuena, 2007; Pontes et al., 2013). Por tanto, creemos que para profundizar en el estudio de la consistencia interna de las concepciones sobre la evaluación de los futuros docentes sería necesario realizar nuevas investigaciones en las que se utilicen instrumentos y técnicas de carácter cuantitativo.

Como conclusión principal, podemos destacar que las concepciones de los estudiantes del MPES sobre la evaluación se pueden relacionar principalmente con los dos enfoques educativos antagónicos, de carácter tradicional o de carácter innovador (próximo al modelo constructivista), y con otro enfoque intermedio que se deriva de aquellas opiniones en las que aparecen elementos de ambos (Turpo-Gebera, 2011; Halinen et al., 2014). Un número ligeramente mayor de opiniones se relaciona con el enfoque innovador o centrado en el alumno, lo cual nos parece interesante como punto de partida para la formación posterior. Pero también encontramos contradicciones y diferencias de enfoque según la cuestión abordada en cada momento y, por otra parte, pensamos que en esta fase formativa los futuros docentes utilizan una especie de constructivismo idealizado para responder a diversas cuestiones, sin ser plenamente conscientes de la dificultad que supone trasladar este enfoque a la práctica educativa real (Mellado et al., 1999; Contreras, 2010; Pontes et al., 2015). Creemos, en cualquier 
caso, que los resultados de este estudio pueden servir como base para avanzar posteriormente en el estudio del pensamiento docente sobre la evaluación utilizando nuevos instrumentos, que permitan recoger datos de carácter cuantitativo y aplicar técnicas estadísticas de análisis multivariante, con vistas a estudiar la consistencia interna de los modelos didácticos y su evolución a través del proceso de formación inicial docente. En la segunda etapa de este proyecto de investigación trataremos de abordar estos aspectos y también trataremos de recoger datos sobre las opiniones del alumnado del MPES acerca del proceso formativo desarrollado en el aula.

\section{Referencias}

Abell, S. K. (2007). Research on science teacher knowledge. En S. K. Abell y G. Lederman (Eds.), Handbook of research on science education (pp. 1105-1149). Londres: Lawrence Erlbaum Associates.

Borko, H. (1997). New forms of classroom assessment: Implications for staff development. Theory into Practice, 36(4), 231-238. doi:10.1080/00405849709543773

Brown, G. T., Lake, R. y Matters, G. (2011). Queensland teachers' conceptions of assessment: The impact of policy priorities on teacher attitudes. Teaching and Teacher Education: An International Journal of Research and Studies, 27(1), 210-220. doi:10.1016/j.tate.2010.08.003.

Buendía, L., Carmona, M., González, D. y López, R. (1999). Concepciones de los profesores de educación secundaria sobre evaluación. Educación XX1, 2(1), 125-154. doi:10.5944/educxx 1.2.0.377.

Carvajal, E. y Gómez, M. (2002). Concepciones y representaciones de los maestros de secundaria y bachillerato sobre la naturaleza, el aprendizaje y la enseñanza de las ciencias. Revista Mexicana de Investigación Educativa, 7(16), 577-602.

Contreras, A. (2010). Las creencias y actuaciones curriculares de los profesores de ciencias de Secundaria de Chile. (Tesis doctoral). Universidad Complutense de Madrid.

De Vries, W. (2015). Las múltiples facetas de la evaluación educativa. Revista Mexicana de Investigación Educativa, 20(66), 679-683.

Dixon, H. y Haigh, M. (2009). Changing mathematics teachers' conceptions of assessment and feedback. Teacher Development, 13(2), 173-186. doi:10.1080/13664530903044002.

Fuentes, M. J., García, S. y Martínez, C. (2009). ¿En qué medida cambian las ideas de los futuros docentes de Secundaria sobre qué y cómo enseñar, después de un proceso de formación? Revista de Educación, 349, 269-294.

García-Carmona, A. (2013). Educación científica y competencias docentes: Análisis de las reflexiones de futuros profesores de Física y Química. Revista Eureka sobre Enseñanza y Divulgación de las Ciencias, 10(Núm. Extraordinario), 552-567.

Halinen, K., Ruohoniemi, M., Katajavuori, N. y Virtanen, V. (2014). Life science teachers' discourse on assessment: A valuable insight into the variable conceptions of assessment in higher education. Journal of Biological Education, 48(1), 16-22. doi:10.1080/002 19266.2013.799082.

Hernández, F. y Maquilón, J. (2010). Las concepciones de la enseñanza. Aportaciones para la formación del profesorado. Revista Electrónica Interuniversitaria de Formación del Profesorado, 13(3), 17-25.

Hernández, L. M. (2010). Evaluar para aprender: Hacia una dimensión comunicativa, formativa y motivadora de la evaluación. Enseñanza de las ciencias, 28(2), 285-290. 
Hewson, P. W. (1993). Constructivism and reflective practice in science teacher education. En M. L. Montero y J. M. Vez (Eds.), Las didácticas específicas en la formación del profesorado (pp. 259-275). Santiago de Compostela: Tórculo.

Meirink, J., Meijer, P., Verloop, N. y Bergen, T. (2009). Understanding teacher learning in secondary education: The relations of teacher activities to changed beliefs about teaching and learning. Teaching and teacher education, 25(1), 89-100. doi: 10.1016/j.tate.2008.07.003

Mellado, V., Blanco, L. y Ruiz, C. (1999). Aprender a enseñar ciencias experimentales en la formación inicial de profesorado. Badajoz: ICE de la Universidad de Extremadura.

Monteagudo Fernández, J., Molina Puche, S. y Miralles Martínez, P. (2015). Opiniones sobre evaluación de los profesores de segundo ciclo de ESO de Geografía e Historia en España: El caso de la Región de Murcia. Revista Mexicana de Investigación Educativa, 20(66), 737761.

Oliva, J. M. (2008). Metodología y recursos educativos: Diseño de materiales didácticos y actividades de aprendizaje. En A. Pontes [Coord.], Aspectos generales de la formación psicopedagógica del profesorado de educación secundaria. (pp. 193-216). Córdoba: Servicio de Publicaciones de la $\mathrm{UCO}$.

Perales, F. J. (2014). La reforma de la formación inicial del profesorado de ciencias de secundaria: Propuesta de un diseño del currículo basado en competencias. Enseñanza de las Ciencias, 32(1), 9-28. doi:10.5565/rev/ensciencias.898.

Pontes, A. y Poyato, F. J. (2014). El enfoque reflexivo en la formación inicial del profesorado de secundaria: Motivaciones por la docencia. Cuadernos de Pedagogía, 445, 34-37.

Pontes, A., Poyato, F. J. y Oliva, J. M. (2015). Concepciones sobre el aprendizaje en estudiantes del máster de profesorado de enseñanza secundaria del área de ciencia y tecnología. Profesorado: Revista de Currículum y Formación del Profesorado, 19(2), 225-243.

Pontes, A., Serrano, R. y Poyato, F. J. (2013). Concepciones y motivaciones sobre el desarrollo profesional docente en la formación inicial del profesorado de enseñanza secundaria. Revista Eureka sobre Enseñanza y Divulgación de las Ciencias, 1O(Núm. Extraordinario), 533-551. doi:10498/15612.

Pontes, A., Serrano, R., Muñoz, J. M. y López, I. (2011). Innovación educativa sobre aprendizaje colaborativo con Cmap Tools en la formación inicial docente. Revista Iberoamericana de Evaluación Educativa, 4(2), 136-154.

Porlán, R. y Martín, R. (2006). ¿Cómo progresa el profesorado al investigar problemas prácticos relacionados con la enseñanza de la ciencia? Alambique: Didáctica de las Ciencias Experimentales, 48, 92-99.

Porlán, R., Rivero, A. y Martín del Pozo, R. (1997). Conocimiento profesional y epistemología de los profesores (I): Teoría, métodos e instrumentos. Enseñanza de las Ciencias, 15(2), 155-171.

Prieto, M. y Contreras, G. (2008). Las concepciones que orientan las prácticas evaluativas de los profesores: Un problema a develar. Estudios Pedagógicos, 34(2), 245-262. doi:10.4067/s0718-07052008000200015.

Remesal, A. (2011). Primary and secondary teachers' conceptions of assessment: A qualitative study. Teaching and Teacher Education: An International Journal of Research and Studies, 27(2), 472-482. doi:10.1016/j.tate.2010.09.017.

Rodríguez, G., Gil, J. y García, E. (1996). Metodología de la investigación cualitativa. Málaga: Aljibe. 
Segers, M. y Tillema, H. (2011). How do Dutch secondary teachers and students conceive the purpose of assessment? Studies in Educational Evaluation, 37(1), 49-54. doi:10.1016/j.stueduc.2011.03.008.

Serrano, R. y Pontes, A. (2015). Nivel de desarrollo de las competencias y objetivos generales del Máster formación del profesorado de enseñanza secundaria. Perfiles Educativos, $37(150), 39-55$.

Serrano, R. y Pontes, A. (2016). Representación del conocimiento sobre la identidad profesional docente en la formación inicial del profesorado. En R. Serrano, M. E. Gómez y C. A. Huertas (Eds), La educación sí que importa en el siglo XXI. (pp. 115-128). Madrid: Síntesis.

Sethusha, M. J. (2013). A vision of improvement of learning: South African teachers' conceptions of classroom assessment. Perspectives in Education, 31(2), 14-21.

Solís, E., Porlán, R. y Rivero, A. (2012). ¿Cómo representar el conocimiento curricular de los profesores de ciencias y su evolución? Enseñanza de las Ciencias, 30(3), 9-30.

Turpo-Gebera, O. W. (2011). Concepciones y prácticas evaluativas de los docentes del área curricular de ciencias en las instituciones de enseñanza públicas de educación secundaria. Revista Iberoamericana de Evaluación Educativa, 4(2), 213-233.

Valbuena, E. O. (2007). El conocimiento didáctico del contenido biológico. Estudio de las concepciones disciplinares y didácticas de futuros docentes de la Universidad Pedagógica Nacional (Colombia). (Tesis doctoral). Universidad Complutense de Madrid.

Vergara, C. E. (2012). Análisis de las concepciones de evaluación del aprendizaje de docentes destacados de Educación Básica. Revista Iberoamericana de Evaluación Educativa, 5(3), 249273.

Wang, J. R., Kao, H. L. y Lin, S. W. (2010). Preservice teachers' initial conceptions about assessment of science learning: The coherence with their views of learning science. Teaching and Teacher Education: An International Journal of Research and Studies, 26(3), 522-529. doi:10.1016/j.tate.2009.06.014 